\title{
Modelling optical spectra and obtaining information on parameters and features of semiconductor structures
}

\author{
V.A. Vasiljev \\ Penza State University, Microelectronics Department, 40 Krasnaya st., 440017 Penza, Russia \\ E-mail:paspen@rambler.ru
}

\begin{abstract}
Problems of semiconductor optical spectrum identification are considered in the paper. Some models for description of optical reflection spectra in semiconductors and semiconductor structures are presented. Shown is the possibility to obtain information upon various parameters and characteristics of semiconductors and their structures by fitting theoretical spectra to experimental data. The estimation of validity and accuracy of obtained information is given.
\end{abstract}

Keywords: semiconductors, optical reflection spectra, semiconductor structures.

Paper received 06.03.02; revised manuscript received 28.06.02; accepted for publication 10.12.02.

Measurement and registration of spectra in each specific case have their own peculiarities [1-7]. Amongst known spectroscopic methods, methods of reflection, transmission and photoconductivity are most often used [4-7]. For spectrum measurements applied are diffraction spectrometers and interferometers of various kinds. The essential progress in the development of spectroscopy is connected with Fourier spectrometers [8-10].

Improvement of methods and facilities of optical spectroscopy is highly actual and significant problem. Its solution cannot be reduced only to tasks of perfecting, modernizing and revising instruments in the hardware part. This problem is complex and depends on instruments, algorithms of processing and methods of spectrum analysis. Certainly, the knowledge of regularities in spectrum changes and methods of spectrum analysis allows finding, registering, as well as producing an evaluation of qualitative and quantitative features of an object under investigation. Combining the optical spectrum methods of measurements with methods of extraction, processing and analysis of information opens broad possibilities in studying different solids.

Within the range of the wavenumbers from 10 up to $1000 \mathrm{~cm}^{-1}$ in optical reflection spectra of semiconductors and semiconductor structures observed are bands of residual rays caused by lattice and plasma vibrations, the latter being oscillations of free charge carriers: electrons or holes. Besides, in optical reflection spectra of film structures, interference phenomena can be pronounced.

In the Fig. 1 shown is the reflection spectrum of the binary semiconductor - gallium arsenide ( $n$-GaAs) with the low concentration of free electrons $\left(n \sim 10^{16} \mathrm{~cm}^{-3}\right)$.
Within the range of wavenumbers from 250 up to $300 \mathrm{~cm}^{-1}$ a band of residual rays (lattice vibrations) is clearly pronounced, while the weak plasma reflection edge can be observed in the range between 20 to $50 \mathrm{~cm}^{-1}$. In the Fig. 1 arrows show frequencies of transversal (TO) and longitudinal (LO) optical phonons.

Identification of the Fig. 1 type spectra and obtaining information on optical phonon frequencies and plasma reflection frequency is usually made visually on the base of knowledge about similar material spectra as well as physical processes caused by optical radiation. Reflection spectra of the film semiconductor structures, espe-

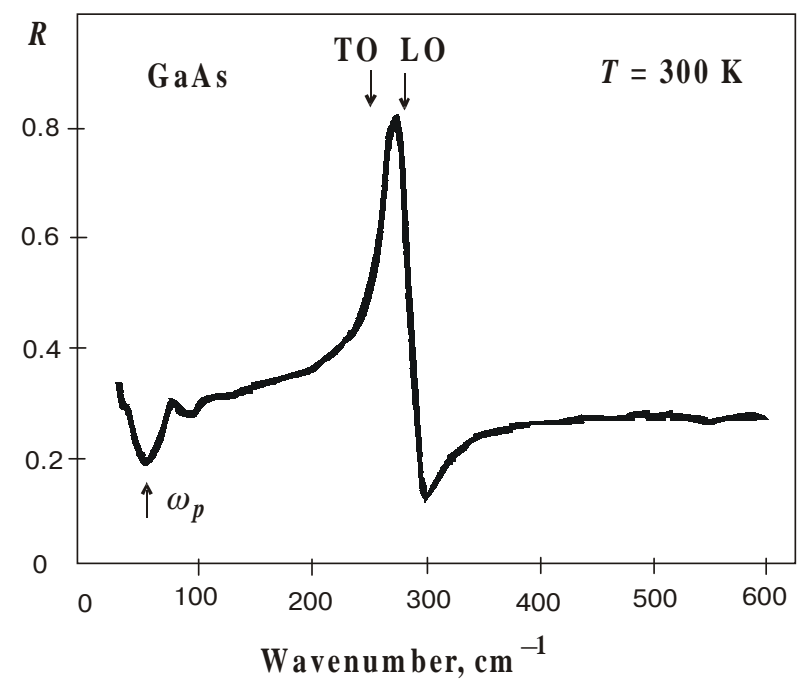

Fig. 1. Optical reflection spectrum of $n$-GaAs. 


\section{V.A. Vasiljev: Modelling optical spectra and obtaining information on parameters...}

cially of multicomponent solid solutions, have more complex appearance and are sufficiently difficult to be identified visually, sometimes, it seems impossible because of superposition with phonon and plasma fluctuations.

Along with it, optical reflection spectra of semiconductors and semiconductor structures can be created by the way of modeling. Tailoring theoretical spectra under experimental data turns out to be possible to determine important parameters and features of semiconductors: plasma frequency $\omega_{p}$, transverse optical phonon frequency $\omega_{T}$, concentration $n$ and mobility $\mu$ of free carriers. Besides, spectra of long-wave optical reflection enable one to determine a thickness of semiconductor and dielectric layers.

Dielectric permeability model. When studying longwave excitations, a crystal can be considered as continuos medium. The macroscopic theory of long-wave optical lattice vibrations in ionic crystals was developed for the first time by Juan Kun [11]. It is based on assumption that longwave vibrations in ionic crystals, containing two ions in each elementary cell, connected with a relative displacement of positive and negative ion sublattices. Consideration of the motion equation for longitudinal and transverse ion vibrations allows to get the simple relation between frequencies of longitudinal $\omega_{L}$ and transversal $\omega_{T}$ vibrations: $\omega_{L}^{2}=\frac{\varepsilon_{S}}{\varepsilon_{\infty}} \omega_{T}^{2}$, where $\varepsilon_{S}-$ steady-state dielectric permeability, $\stackrel{\infty}{\varepsilon}_{\infty}$ - dielectric permeability at an infinitly large frequency.

According to the dielectric permeability model (taking into account non-harmonic effects) developed by Juan Kun, the refraction index could be determined by the formula:

$$
n(\omega)=\sqrt{\varepsilon(\omega)}=\left(\varepsilon_{\infty}+\frac{\left(\varepsilon_{s}-\varepsilon_{\infty}\right) \omega_{T}^{2}}{\omega_{T}^{2}-\omega^{2}-i \gamma \omega}\right)^{\frac{1}{2}},
$$

where $\gamma$ is the decay parameter; $\omega$-a current frequency.

If a photon energy is less then the forbidding gap, then interaction of electromagnetic radiation with free carriers becomes essential. The refraction index in this case can be determined by the formula [12]:

$$
n(\omega)=\sqrt{\varepsilon(\omega)}=\left(\varepsilon_{\infty}-\frac{\varepsilon_{\infty} \omega_{p}^{2}}{\omega \cdot\left(\omega+\frac{i}{\tau}\right)}\right)^{\frac{1}{2}},
$$

where $\omega_{p}$ is a plasma frequency; $\tau=\frac{m^{*} \mu}{e}, m^{*}-$ an effective mass of carriers; $\mu-$ a mobility of carriers; $e-$ the charge of the electron.

Taking into account contributions of lattice (1) and plasma vibrations (2) the refraction index can be determined by the formula [4]:

$$
\begin{aligned}
& n(\omega)=\sqrt{\varepsilon(\omega)}= \\
& =\left\{\varepsilon_{\infty}-\frac{\varepsilon_{\infty} \omega_{p}^{2}}{\omega(\omega+i / \tau)}+\sum_{j} \frac{s_{j} \omega_{T j}^{2}}{\omega_{T j}^{2}-\omega^{2}-i \omega \gamma_{j}}\right\}^{\frac{1}{2}},
\end{aligned}
$$

where $\varepsilon_{\infty}$ is a high frequency dielectric permeability; $\omega_{p}$ - plasma frequency; $\omega_{T j}$-a frequency of $j$-transversal optical phonons; $\omega$-a current frequency; $s_{j}-$ a power of the $j$-oscillator; $\tau-$ a time of relaxation; $\gamma_{j}-$ a decay parameter. In the case of a solid solution, the high-frequency dielectric permeability $\varepsilon_{\infty}$ can be determined by the linear interpolation of a respective component values. Power of possible oscillators can be calculated by the formula: $s_{j}=\left(\varepsilon_{s j}-\varepsilon_{\infty j}\right) x_{j}$, where $\varepsilon_{\infty j}, \varepsilon_{s j}-$ high frequency and low frequency dielectric permeabilities; $x_{j}$ - molar share of the component.

Modeling of optical reflection spectra of semiconductors and semiconductor structures can be based on generalized models of the refraction index (dielectric permeability) (3).

Matrix formalism in optical reflection spectrum calculations for semiconductor structures. Determination of the reflection index of non-homogeneous semiconductor structures is required in some cases. To describe of optical reflection spectrum of the semiconductor structures consisting of an epitaxial layer on a substrate, one can use the single-layer model. In this case the reflection index from the substrate side is [3]:

$$
R=\left|\frac{(1-n)+(1+n)\left[\left(n-n_{s}\right) /\left(n+n_{s}\right)\right] \cdot \exp (i 4 \pi \sigma h)}{(1+n)+(1-n)\left[\left(n-n_{s}\right) /\left(n+n_{s}\right)\right] \cdot \exp (i 4 \pi \sigma h)}\right|^{2}
$$

where $n, n_{s}$ - indexes of layer and substrate refraction, respectively, $h-$ a layer thickness, $\sigma-$ a wavenumber, $i=\sqrt{-1}$.

For modeling the optical reflection spectra of semiconductors and semiconductor structures, the matrix method is the most suitable. The matrix method as applied to dielectric layers is described in [13]. This method allows to take into account non-homogeneity of solid structures by splitting them on separate uniform layers and describing each layer by a characteristic matrix.

If a multi-layer structure consists of $N$ layers (see Fig. 2), the matrix $M$ for the area, comprised between $z=0$ and $z=h$, equals to a product of the matrixes $M_{j}$ for these layers:

$$
M=\prod_{j=1}^{N} M_{j}
$$

In the case of a uniform layer $\left(n_{1}=n_{2}=n\right)$ the characteristic matrix is as follows:

$M=\left[\begin{array}{ll}\cos \beta n & (-i / n) \sin \beta n \\ -i n \sin \beta n & \cos \beta n\end{array}\right]$,

where $\beta=2 \pi \sigma h, \sigma-$ a wavenumber, $i=\sqrt{-1}$. 


\section{V.A. Vasiljev: Modelling optical spectra and obtaining information on parameters...}

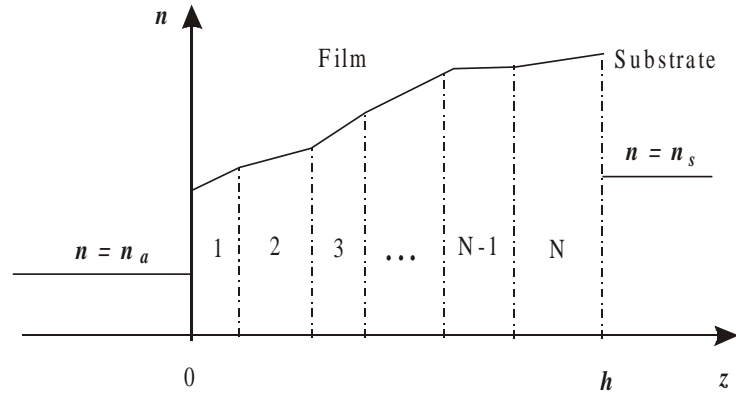

Fig. 2. Schematic of the refraction index profile for the multilayer film structure.

In practice, often used are layers having gradient of refraction index, the value of which can reflect epitaxial growth conditions, processes of diffusion or created intentionally to provide required structure features. Hyperbolic distribution of the refraction index can be used for such structure description:

$$
n(z)=\frac{n_{1} n_{2}}{\left[n_{2}-\left(n_{2}-n_{1}\right) \frac{z}{h}\right]},
$$

where $n(z)$ is a refraction index, $n_{1}$ and $n_{2}$ - values of refraction index at layer boundaries, $h$ - its thickness. This distribution is rather suitable, because under a small value of $\frac{\left(n_{2}-n_{1}\right)}{n_{1}}$ it is practically linear and allows, when using a matrix formalism, to write a characteristic matrix of the layer in the following analytical form:

$M=\left[\begin{array}{ll}\left(\frac{n_{2}}{n_{1}}\right)^{\frac{1}{2}}\left(\cos \alpha+\frac{1}{2 m} \sin \alpha\right) & i \beta \frac{\left(n_{1} \cdot n_{2}\right)^{\frac{1}{2}}}{m\left(n_{2}-n_{1}\right)} \sin \alpha \\ i \beta \frac{\left(n_{1} \cdot n_{2}\right)^{\frac{3}{2}}}{m\left(n_{2}-n_{1}\right)} \sin \alpha & \left(\frac{n_{1}}{n_{2}}\right)^{\frac{1}{2}}\left(\cos \alpha-\frac{1}{2 m} \sin \alpha\right)\end{array}\right]$,

where $\alpha=m \cdot \ln \left(\frac{n_{1}}{n_{2}}\right), m^{2}=\left[\frac{\beta \cdot n_{1} \cdot n_{2}}{\left(n_{2}-n_{1}\right)}\right]^{2}-\frac{1}{4}, \beta=2 \pi \sigma h$, $\sigma$-wavenumber, $h$-layer thickness.

The reflection coefficient as a function of an electric field strength is expressed through the matrix elements $m_{i k}$ :
$R=\left|\frac{\left(m_{11}+m_{12} \cdot n_{s}\right) n_{a}-\left(m_{21}+m_{22} n_{s}\right)}{\left(m_{11}+m_{12} n_{s}\right) n_{a}+\left(m_{21}+m_{22} n_{s}\right)}\right|^{2}$,

where $n_{s}-$ a substrate refraction index.

Spectrum modeling. In the Fig. 3, the solid line presents reflection spectra of the binary semiconductors InSb $\left(n \sim 10^{16} \mathrm{~cm}^{-3}\right)$, InAs $\left(n \sim 2.6 \cdot 10^{16} \mathrm{~cm}^{-3}\right)$ and InP $\left(n \sim 10^{16} \mathrm{~cm}^{-3}\right)$, in long-wave IR band. In these spectra, the bands of residual rays at 185, 220 and $310 \mathrm{~cm}^{-1}$, as well as plasma reflection near $100 \mathrm{~cm}^{-1}$ are clearly pronounced.

Optical reflection spectra shown in the Fig. 3 are well described using the single oscillator dielectric permeability model, according to which refraction index is expressed by the formula (3), at the number of oscillators $j=1$. Theoretical spectra were fitted to the experimental ones till achieving the best coincidence. Values $\omega_{P}, \omega_{T}$, $\tau, S=\varepsilon_{S}-\varepsilon_{\infty}$ and $\gamma$ were used as variable parameters. Parameter values of samples 1,2 and 3 (see Fig.3), determined as a result of fitting the theoretical spectra to experimental data, represented in the Table 1.

In the Fig. 4 shown are the reflection spectra of nInAs samples having different free carriers concentrations. The sample 2 has larger free carrier concentration, than the sample 1. Influence of free carriers on the reflection spectrum is graphically illustrated. The edge of plasma reflection with increasing concentration of free carriers is displaced to short waves (greater wavenumbers).

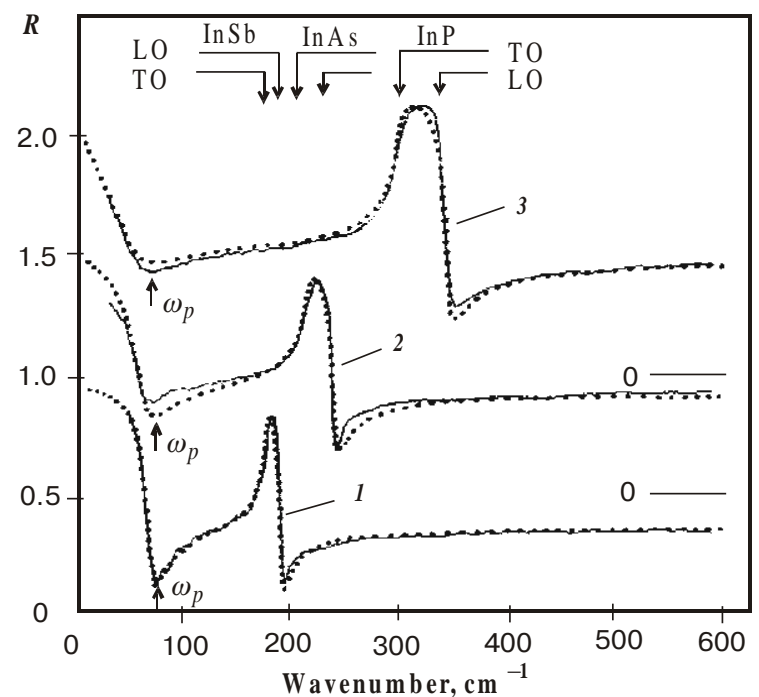

Fig. 3. Reflection spectra of $n-I n S b, n-I n A s$ and $n-I n P$ at $300 \mathrm{~K}$. Lines show experimental data, dots - calculated ones. Spectra 2 and 3 are shifted along the vertical axis. Origins are marked to the right.

Table 1. Values of parameters for three samples of semiconductor compounds

\begin{tabular}{llllllll}
\hline \hline Sample & $\varepsilon_{\infty}$ & $\varepsilon_{s}$ & $\omega_{T}, \mathrm{~cm}^{-1}$ & $S$ & $\gamma, \mathrm{cm}^{-1}$ & $\omega_{p}, \mathrm{~cm}^{-1}$ & $\tau, \mathrm{cm}$ \\
\hline $1-n$-InSb & 15.7 & 17.9 & 180 & 2.2 & 4.0 & 78 & 0.08 \\
\hline $2-n$-InAs & 11.8 & 14.6 & 219 & 2.8 & 8.0 & 73 & 0.04 \\
\hline $3-n$-InP & 9.6 & 12.6 & 306 & 3.0 & 5.5 & 65 & 0.02 \\
\hline \hline
\end{tabular}




\section{V.A. Vasiljev: Modelling optical spectra and obtaining information on parameters...}

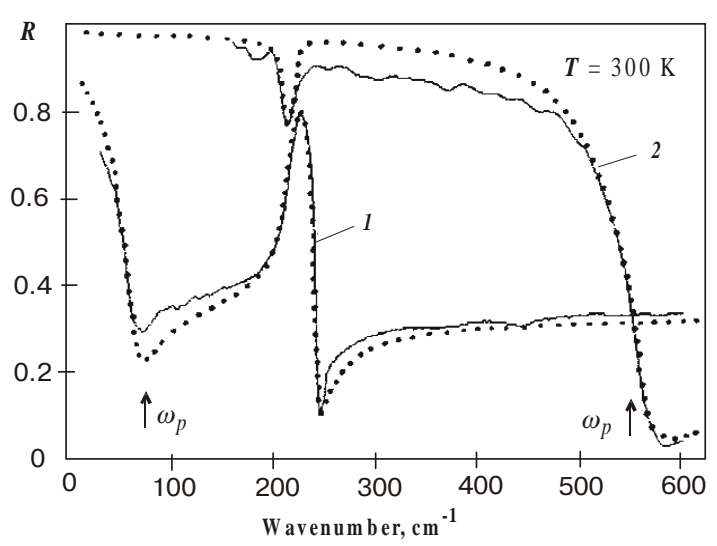

Fig. 4. Reflection spectra of $n-I n A s$ for two values of free charge carrier concentrations: $1-n \approx 2.6 \cdot 10^{16} \mathrm{~cm}^{-3}\left(\omega_{p}=73 \mathrm{~cm}^{-1}\right) ; 2-$ $n \approx 2.6 \cdot 10^{18} \mathrm{~cm}^{-3}\left(\omega_{p}=550 \mathrm{~cm}^{-1}\right)$. Line - experiment, dots calculation

In the Fig. 5 depicted are changes of minimum positions in the reflection spectrum depending on plasma frequencies $\omega_{p}$. Reduction of the relaxation time $\tau$ with increase $\omega_{p}$ taken into account in all calculations. Change was supposed to be linear, but numeric values were based on data obtained when analysing experimental curves of Fig. 4. Strong interaction (coupling) between LO phonons and plasmons appears in the case of $\omega_{p} \approx \omega_{\mathrm{LO}}$. Herewith, lower minimum is displaced below $\omega_{\mathrm{LO}}$ (refer to the curve

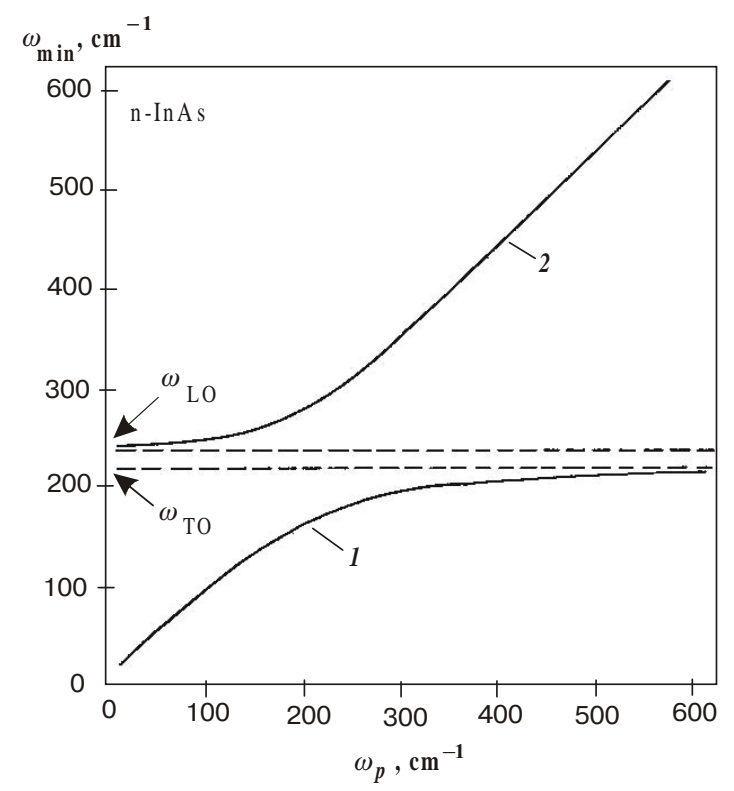

Fig. 5. Change of minima positions in n-InAs spectrum in dependency on the plasma frequency $\omega_{p}$. $l$ in the Fig. 5), while the upper one above $\omega_{\mathrm{LO}}$ (the curve 2 in the Fig. 5).

In practice often used are multi-layer structures and layers having a gradient of composition, concentration and mobility of free carriers. In the Fig. 6, optical reflection spectra of the epitaxial structure p$\mathrm{InAs}_{0.63} \mathrm{Sb}_{0.12} \mathrm{P}_{0.25}: \mathrm{Zn} / \mathrm{n}$-InAs are shown. The main feature of these reflection spectra is that they cannot be described by means of single-layer model because of the deep penetration of zinc into the substrate during the diffusion process. To describe the spectrum of optical reflection, we used the two-layer model. The refraction index was calculated by the formula (3) at the number of oscillators $j=3$. The characteristic matrixes of layers and substrates were determined by the formula (6), the characteristic matrix of the structure - by the formula (5), the reflection coefficient - by the formula (9). Values of layer parameters, determined due to procedures of fitting the theoretical spectra to experimental data, are represented in the Table 2 .

The first figure in the index (see Table 2) indicates a layer number, the second one-an oscillator number (for $\gamma$ ). Notice that first from the surface layer is a solid solution, while the second layer is formed in the substrate due to diffusion of $\mathrm{Zn}$ impurity. It is ascertained that the first layer thickness is $h_{1}=2.2 \mu \mathrm{m}$, but the second one $h_{2}=18 \mu \mathrm{m}$. The parameter $h_{2}$ defines a period of oscillation in the part of the spectrum below $200 \mathrm{~cm}^{-1}$. These oscillations are caused by interference in the layer between the space charge area and epitaxial layer. By the parameter $h_{\Sigma}=h_{1}+h_{2}$ it is possible to evaluate the position of the $p$ $n$ transition in this structure.

Some better coincidence between theoretical and experimental results (as compared to Fig. 6, approximately by $5 \%$ ) is reached when using the two-layer model with gradient layers, in which the hyperbolic distribution (7) and characteristic matrix (8) are used.

The quality of information obtained using the calculating models of optical spectra can be judged considering the validity and accuracy of determined parameters of semiconductor structures. On the base of parameters of calculating models, it is possible to quantitatively evaluate different semiconductors features.

Evaluation of validity and accuracy. Validity and accuracy of parameters obtained from calculating models, were estimated using sensitivity to such parameters of the layer $\omega_{n}, \tau, x$ and $h$ taking as an example the $p$ $\mathrm{InAs}_{0.63} \mathrm{Sb}_{0.12} \mathrm{P}_{0.25} / n$-InAs structure. The calculated model has sufficiently high sensitivity to changes of plasma frequencies $\omega_{p}$. Moreover, it is observed practically a linear dependency. So, with changing $\omega_{p}$ by $10 \%$, the maximum value of the reflection coefficient $R$ varies by ap-

Table 2. Parameters of layers for the semiconductor structure $p$-InAs ${ }_{0.63} \mathrm{Sb}_{0.12} \mathrm{P}_{0.25}: \mathrm{Zn} / n$-InAs

\begin{tabular}{lcccccccc}
\hline \hline$\omega_{p 1}, \mathrm{~cm}^{-1}$ & $\omega_{p 2}, \mathrm{~cm}^{-1}$ & $\tau_{1} \cdot 10^{3}, \mathrm{~cm}$ & $\tau_{2} \cdot 10^{3}, \mathrm{~cm}$ & $\gamma_{11}, \mathrm{~cm}^{-1}$ & $\gamma_{12}, \mathrm{~cm}^{-1}$ & $\gamma_{13}, \mathrm{~cm}^{-1}$ & $h_{1}, \mu \mathrm{m}$ & $h_{2}, \mu \mathrm{m}$ \\
\hline 220 & 55 & 2.0 & 0.11 & 16 & 25 & 27 & 2,2 & 18 \\
\hline \hline
\end{tabular}




\section{V.A. Vasiljev: Modelling optical spectra and obtaining information on parameters...}

proximately $10 \%$, too. It should be noted that the largest change of the reflection coefficient takes place in the range of lattice vibration frequencies and is connected with plasmon-phonon interaction. Analysis of other parameter deviation shows that the time of relaxation $\tau$ weakly influences upon the reflection coefficient $R$, and when $\tau$ changes by $50 \%$, the reflection coefficient $R$ is changed not more than by $8 \%$. The model is sufficiently well sensitive to changing the parameters $h$ and $x$. The change of $h$ by $50 \%$ results in the change of the reflection coefficient $R$ up to $15 \%$, but $x$ by $10 \%$ - to changing the reflection coefficient $R$ up to $25 \%$. If the lower limit of sensitivity is taken as $\sim 3 \%$, we can get estimates of relative inaccuracies of determined parameters. The relative inaccuracy of parameter determination for a semiconductor layer is summarized represented in the Table 3.

Evaluation of free carrier concentrations and mobilities. Fitting parameters obtained from the calculating models and accumulated in tables 2 and 3, enabled us to eventually determine such important features of semiconductors as a concentration of free charge carriers and their mobility: $n=\frac{\omega_{p}^{2} m^{*} \varepsilon_{0} \varepsilon_{\infty}}{e^{2}}$ and $\mu=\frac{\tau \cdot e}{m^{*}}$. Here, $\varepsilon_{0}$ is a dielectric constant, $m^{*}$ - effective mass of charge carriers; $\mu$-mobility of charge carriers; $e$-charge of the electron. Values $n$ and $\mu$ obtained from optical measurements sufficiently well coincide with $n$ and $\mu$ values determined using the Hall e.m.f. measurements.

Evaluation of the penetration depth for electromagnetic radiation. The formula $I(x)=I_{0} \exp (-\alpha x)$ is known in physics as the Bouguer-Lambert-Beer law, where

Table 3. Relative inaccuracy in determined parameters of the semiconductor layer

\begin{tabular}{lcc}
\hline Parameter & $\begin{array}{c}\text { Relative } \\
\text { inaccuracy }\end{array}$ & Basic value \\
\hline$\omega_{p}$ & 0.03 & $210 \mathrm{~cm}^{-1}$ \\
\hline$\tau$ & 0.5 & $0.03 \mathrm{~cm}$ \\
\hline$h$ & 0.15 & $2.4 \mu \mathrm{m}$ \\
\hline$x$ & 0.05 & 0.25 \\
\hline \hline
\end{tabular}

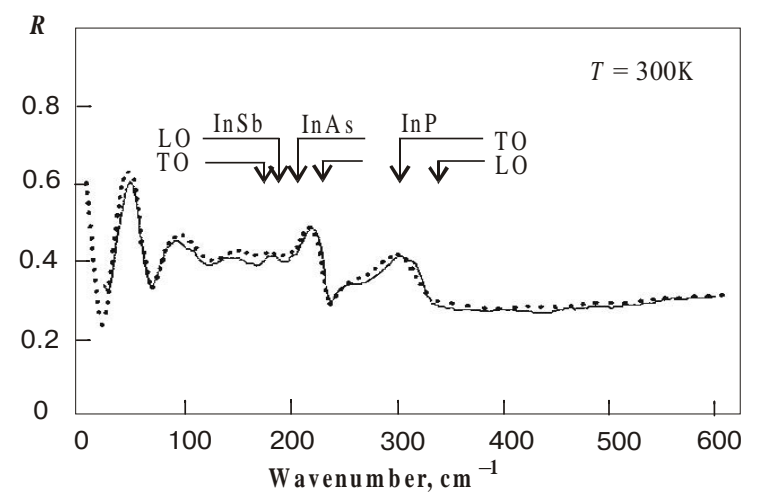

Fig. 6. Reflection spectrum of the epitaxial structure $p$ $\mathrm{InAs}_{0.63} \mathrm{Sb0}{ }_{.12} \mathrm{P}_{0.25}: \mathrm{Zn} / n$-InAs. Line - experimental, dots - calculation. $\alpha=2 \frac{\omega \cdot n^{\prime \prime}}{c}$ is an absorption coefficient, $I_{0}$-intensity of light entering through a surface into a sample. It follows from the formula that the value inverse to the absorption coefficient $\alpha^{-1}$ is numerically equal to the layer thickness that provides weakening the intensity of passing light by $e$ times. The depth of electromagnetic penetration can be evaluated using the formula:

$d=\frac{1}{2 \pi \sigma n^{\prime \prime}(\omega)}$,

where $n^{\prime \prime}(\omega)$ is the imaginary part of the refraction index, $\sigma$ - wavenumber.

In the Fig. 7 illustrated are dependencies of the penetration depth of electromagnetic radiation on the frequency for different values $\omega_{n}$ calculated using the formula (10). The imaginary part of the refraction index $n^{\prime \prime}(\omega)$ was determined by the formula (3). The lowest value of the penetration depth takes place near $\omega_{T}$. With increase of the plasma frequency the penetration depth decreases. So, under $\omega_{p}=550 \mathrm{~cm}^{-1}$ (that corresponds to $n \sim 10^{18} \mathrm{~cm}^{-3}$ ), curve 1 (Fig.7), the penetration depth does not exceed some micrometers.

As seen from the presented material, optical reflection of semiconductors and semiconductor structures is rather informative. The developed way of modeling the spectra of optical reflection of semiconductor structures allows to take into account an arbitrary amount of layers, both uniform and the gradient ones. The method is applicable for the broad class of semiconductor structures on the base of $\mathrm{A}^{3} \mathrm{~B}^{5}$ solid solutions and other compounds.

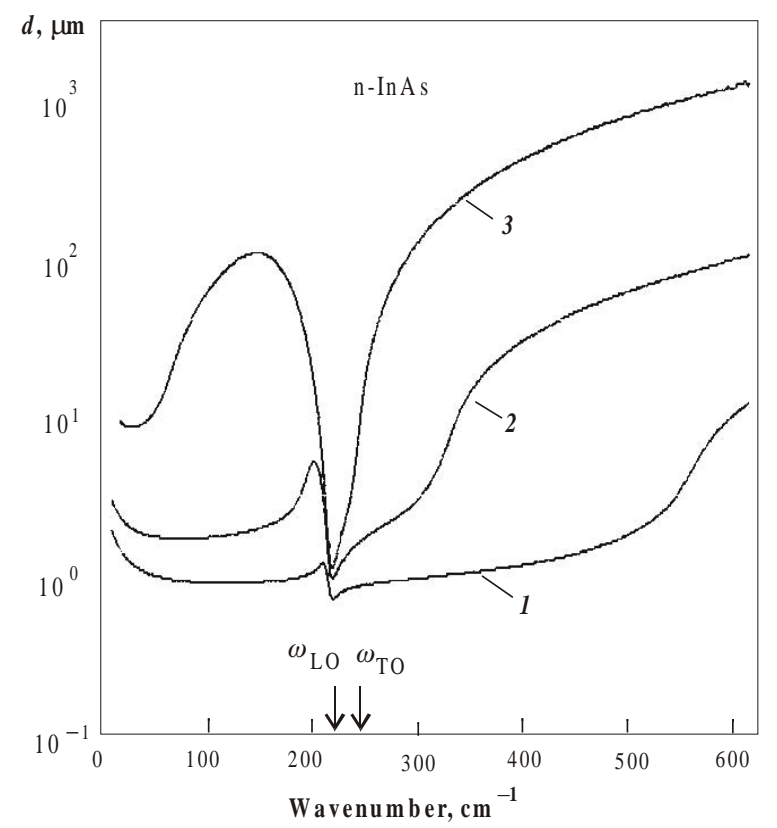

Fig. 7. Depth of optical emission penetration into $n$-InAs: $1-$ $\omega_{p}=550 \mathrm{~cm}^{-1} ; 2-\omega_{p}=300 \mathrm{~cm}^{-1} ; 3-\omega_{p}=75 \mathrm{~cm}^{-1}$.

$S Q O, 5(3), 2002$ 


\section{V.A. Vasiljev: Modelling optical spectra and obtaining information on parameters...}

\section{References}

1. Vasiljev V.A. Optical spectrum methods of shaping information resources of solid state structures. In the book: XXVII International conference "Information technologies in the science, formation, telecommunications and business". Yalta, Gurzuf, 2000, p.182-186.

2. Vasiljev V.A. Registration and information handling in optical spectroscopy for shaping the information resources of solid state structures. In the book: International symposium "Reliability and quality'2001". Penza, 2001, p.255-259.

3. Zinger G.M., Ipatova I.P., Ryskin A.I. Optical characteristics four components solid dissolve on the base of join in the field of lattice and plasma oscillation. // Physics and techniques of semiconductors. - 1984. - Vol.18, №.1. - p.24-42

4. Baranov A.N., Vasiljev V.A., Kopylov A.A., Sherstnev V.V./ Optical reflection and determination of epitaxial structure features // Physics and techniques of semiconductors. - 1991., Vol. 25, №.1, p.99-101.

5. Vasiljev V.A., Dyshlovenko P.E., Kopylov A.A., Shakmaev A.A. Long wave Fourier spectroscopy of shallow impurities in GaP. Optoelectronic materials: Collection of scientific works/Leningrad electrotechnical institute by the name of V.I.Ulianov (Lenin). - L., 1989. - №. 414. - p.21-24.
6. Vasiljev V.A. Impurities photoconductivity in solid solutions germanium-silicon. Collection of scientific works/Leningrad electrotechnical institute by the name of V.I.Ulianov (Lenin). - L. 1990. - №.420. - p.17-21.

7. Vasiljev V.A., Dyshlovenko P.E., Kopylov A.A., Lutovich K.L. Impurity photoconductivity of $\mathrm{Ge}-\mathrm{Si}$ epitaxial layers doped by phosphorus. Physics and techniques of semiconductors. - 1990. Vol. 24, № 9. - p.1675-1677.

8. Automated Fourier-spectrometer for low temperature optical measurements in the range of $10-650 \mathrm{~cm}^{-1} /$ Vasiljev V.A., Dyshlovenko P.E., Kopylov A.A., Holodilov A.N.// Instruments and technology of experiment. - 1990. - №5. - p.174-177.

9. Vasiljev V.A., Kopylov A.A., Holodilov A.N. Fourier spectrometer. Author's certificate. USSR. № 1681171, МКИ G $01 \mathrm{~J}$ 3/28 (Bulletin of inventions № 36, 30.09.1991.).

10. Vasiljev V.A., Kopylov A.A., Holodilov A.N. Fourier spectrometer. Author's certificate. USSR. № 1622775, МКИ G $01 \mathrm{~J}$ 3/28 (Bulletin of inventions № 3, 23.01.1991.).

11. Boric M., Juan Kun. Dynamic theory of crystalline lattices. - M., 1958. - 488 P.

12. Animalu A. Quantizing theory of crystalline solids - M.: World, 1981. - 574 P.

13. Jacobson R. - In the book: Physics of thin films. Vol.8/Edited by G.Huss, M.Frankomb and RGofman. - M.: World,1967. - p.91-151. 